\title{
Ocular Delivery of Drugs
}

\author{
Clyde Schultz ${ }^{1,2 *}$ \\ ${ }^{1}$ Department of Biology, University of Calgary, Calgary, Alberta, Canada \\ ${ }^{2}$ Biogram Inc., Ponte Vedra, FL, USA
}

\begin{abstract}
This article discusses the treatment of ocular retinal segment disease that occurs in humans, including treatments other than injection of drugs to the retinal or posterior segment. Many of the drugs used have been shown to be effective in modulating abnormal cellular growth but delivering them to the location of action is challenging. Various new techniques, including implants, contact lens delivery and surgically implanted reservoirs are described.
\end{abstract}

\section{Introduction}

The posterior segment of the human eye is in many respects one of the most remote areas of the human body to treat. This area of the body is fragile and is complex in that there are combinations of nerves, blood vessels and specialized cells, which evolved to provide clear colour vision, one of the major factors in human evolution. Drug delivery to the retinal segment, often referred to as the posterior segment, involves many challenges that drug delivery or use on the anterior segment does not involve. This area is the disease site for both age related macular degeneration (AMD) and diabetic retinopathy $(\mathrm{DM})$. The delivery itself is usually by injection and this procedure is not without risk. Further, the drug must be delivered via a route that contains any number of enzymes designed to destroy the drug being delivered. Newer techniques based on a drug delivery "vehicle" which contains a cargo space much like a suitcase are being explored for potential use to deliver medicament to the posterior segment area where potential treatments are not easily or effectively delivered. The use of implants, contact lenses and refillable reservoirs all have their potential use in this area. The route of inoculation for these may be different, but the purpose is to modulate vascular endothelial growth factor (VEGF) which is responsible for blood vessel encroachment and thus a narrowing of vision or loss of lines of vision as a result of their presence. This paper will explore some broad treatment categories which are being used for the treatment of AMD and DR.

\section{Contact lens treatment}

The prevailing view among medical professionals for decades was that contact lenses could only be used for vision correction and that even though a stable "cargo space" was available it was unsuitable for drug delivery. This was "common knowledge" despite evidence to the contrary. OCUSERT is a medical device/drug delivery system which coupled a contact lens type pouch with pilocarpine in an attempt to treat glaucoma [1]. The product was reported to be uncomfortable and suffered from a tendency of the lenses to "pop" out of the eye, but in those cases where it could be worn, it was successful at controlling elevated intraocular pressure (IOP) [1].

Modern hydrogels have also been used to deliver drugs to the eye. A small clinical study with timolol maleate and brimodium tartrate was successful at modulating intraocular pressure [2]. In this case an etafilcon A hydrogel was passively loaded with both of these drugs. In another study, Epidermal Growth Factor (EGF) released from hydrogel contact lenses was shown to be effective in closing corneal wounds in humans [3]. It was shown by HPLC that neither of the glaucoma drugs or EGF was altered in terms of the molecular structure. Additional experiments in New Zealand White rabbits showed a statistically significant difference between EGF treated and untreated eyes following artificial wounding of the cornea [4]. Other work demonstrated the release kinetics of prednisolone and beclomethasone from different contact lens polymers by HPLC [5]. HPLC was also used to monitor uptake and release of contact lenses passively loaded with prednisolone and the molecules subsequent appearance in the posterior segment of the eyes of New Zealand White rabbits [5]. This work also demonstrated a potential pathway by which the drugs matriculated to the posterior segment-not through the vitreous humor but around the globe of the eye. HPLC analysis of the systemic blood and vitreous humor supported this hypotheses in that none were detected [5].

Other authors have used contact lenses or the materials that compose them to deliver drugs to the posterior segment, but not all treatments have been successful. There is evidence that not all hydrogel polymers will take a given compound into their cargo space [2].

One of the biggest issues with the use of contact lenses or any polymer to treat disease is manufacture. Combination products are comprised of some sort of drug and a carrier, often a polymer. Drugs are susceptible to extremes in heat, making terminal steam sterilization not available as an option to sterilize the product. The carrier which acts as a "cargo space" may be an implant or a contact lens. Most polymers that are formulated into contact lenses or other types of polymer-based products can withstand the heat and pressure of steam sterilization, without being altered in any way that compromises their ability to be worn comfortably, or for vision correction. This cannot be said

Correspondence to: Clyde Schultz, Department of Biology, University of Calgary, Calgary, Alberta, Canada, Tel: 403.220.5278, E-mail: schultzc@ucalgary.ca

Key words: Ocular, Posterior, Drug, Contact Lens, Injection

Received: January 23, 2018; Accepted: March 24, 2018; Published: March 31,2018 
for the drugs that are the actual disease modification agents or active pharmaceutical agents (API). Most of these compounds are susceptible to extremes in temperature and pressure and will degrade rapidly.

The best option for larger scale production of product is to manufacture in an aseptic environment. This is more time consuming and costly but is effective in product manufacture. Costs can be mitigated somewhat if manufacturing is performed outside the United States. However, product quality may be less rigorous than manufacturing done in the United States, Canada or Europe.

\section{Posterior segment injections}

Injections into the posterior segment of the eye have been used effectively since penicillin was first injected into abscesses following cataract surgery. They are typically performed today with a 30 -guage needle conveniently packaged as part of a "kit" which contain the delivery syringe and needle, the drug, or active agent in freeze dried form and a diluent usually saline that is used to rehydrate the drug. This rehydrated drug is then injected into the eye using the syringe. The repetitive nature of the therapy combined with the risk profile and subsequent patient discomfort makes injections a less desirable course of treatment for the eye than for other sorts of systemic disease. However, at this time, this method is considered the Gold Standard for this type of delivery. Avastin and Lucentis are among the biological molecules that are delivered by injection to the posterior segments [68]. They are used in the treatment of AMD.

\section{Ocular implants and reservoirs}

Implants are a current method for delivery of drugs to both anterior and posterior segment of the eye. The wide range of diseases include some glaucoma diagnosis, posterior uveitis, diabetic macular edema, and cytomegalovirus infections secondary to HIV infection are examples of the syndromes these devices were designed to treat. The implants require replacement on different time schedules depending on the drug being delivered and the polymer used as the delivery device. In contrast ocular reservoirs do not necessarily require replacement. Much like the gas tank on a car, these reservoirs require "refilling". An example is the Replenish MicroPump (Replenish, Pasadena, CA). The reservoir is re-filled by transconjunctival injection. This pump is designed to release nanoliter quantities of drug into the ocular space over a period of time. Other systems similar to this for age related macular degeneration and non-infectious uveitis are also in development (ForSight VISION 4).

A variation on implants is the use of Matrix films for drug delivery. A polyethylene oxide based melt-cast matrix system was developed for delivery of hesperetin [9]. Experiments in rabbits have shown that the delivery of the drug to at least some of the retinal space was possible using this approach. Retisert and Ozurdex are more recent examples of implants or inserts that have been used to treat posterior segment disease. Retisert elutes fluocinolone acetonide into the vitreous humor for several years [10]. The 24-month Multicenter Uveitis Steroid Treatment (MUST) trial was conducted to compare the delivery systems plus steroid to the delivery of systemic steroids for the treatment of non-infectious uveitis [11]. Retisert improved visual acuity over the course of the study, but the study also showed an $81 \%$ increase in cataract surgery and $17 \%$ increase in glaucoma in the treatment group. Sangwan and co-workers reported reduced recurrence of uveitis rates among a three-year treatment group as compared to the preceding year of the same group [12].
Ozurdex is an implant which has $0.7 \mathrm{mg}$ dexamethasone as the active agent. This product is used to treat posterior segment uveitis in both adults and children [13]. In a study for treatment of macular edema, $30 \%$ of 412 eyes gained a minimum of 15 letters after 60 days. In another study, $22 \%$ of treated eyes gained at least a 15 letter increase in best corrected visual acuity (BCVA), while 12\% of sham control achieved the same result. However, safety data indicate that Ozurdex may lead to the development of corneal edema.

Inserts include Iluvien which was approved for the treatment of diabetic macular edema in 2014 [14]. Steroid eluting devices are on the market and some of the most common use dexamethasone as the active agent. An insert with dexamethasone has been shown to have a lower associated rate of ocular hypertension than injectable drug [14]. The Port delivery system is a refillable drug delivery device for the treatment of age related macular degeneration.

\section{Conclusion}

The exploration of the use of combination products will continue to gain prominence in the medical community. The systems for delivery of drugs are proving to be as important in disease treatment as the drugs themselves. This is due to the necessity to delivery drugs by more efficient means. In some cases, there is an intellectual property concern, specifically lengthening the time frame for intellectual property protection for a given drug. This may be accomplished by delivery of a drug via the combination route with a medical device. In some respects, the low hanging fruit of drug and biologic delivery have been discovered. As disease syndromes evolve so must the delivery of drugs to the locations of action. This will involve the development of new polymers and new manufacturing strategy to make them suitable for human use.

\section{References}

1. Gale R. US Patent US4190642A.1980.

2. Schultz CL, Poling TR, Mint JO (2009) A medical device/drug delivery system for treatment of glaucoma. Clin Exp Optom 92: 343-348. [Crossref]

3. Holland S, et al. (2012) Wound Healing Using a Medical Device Drug Delivery System. Clinical and Experimental Ophthalmol 40: 662-668.

4. Schultz C, Morck DW (2010) Contact Lenses as a Drug Delivery Device for Epiderma Growth Factor in the Treatment of Ocular Wounds. Clin Exp Optom 93:61-65. [Crossref]

5. Schultz C, Breaux J, Schentag J, Morck D (2011) Drug delivery to the posterior segment of the eye through hydrogel contact lenses. Clin Exp Optom 94:212-218. [Crossref]

6. Arvelo JF, Fromow-Guerra J, Sanchez JG, Maia M, Berrocal MH, et al. (2008) Primary intravitreal bevacizumab for subfoveal choroidal neovascularization in age related macular degeneration: results of the Pan American Collaborative Study Group at 12 months follow-up. Retina 28: 1387-94. [Crossref]

7. Azad R, et al. (2007) Intravitreal bevacizumab in aggressive posterior retinopathy of prematurity. Indian Journal of Ophthalmology 55: 319-22.

8. Solomon SD, Lindsley K, Vedula SS, Krzystolik MG, Hawkins BS (2014) Antivascular endothelial growth factor for age related macular degeneration. Cochrane Database Syst Rev 8:CD005 5139. [Crossref]

9. Adelli GR, Hingorani T, Punyamurthula N, Balguri SP, Majumdar S (2015). Evaluation of topical hesperetin matrix film for back-of-the-eye delivery. Eur J Pharm Biopharm 92: 74-82. [Crossref]

10. Jaffe GJ, Martin D, Callanan D, Pearson PA, Levy B, et al. (2006) Fluocinolone acetonide uveitis study group. Fluocinolone acetonide implant (Retisert) for noninfectious posterior uveitis: thirty four week results of a multicenter randomized clinical study. Ophthalmology 113: 1020-7. [Crossref] 
11. Multicenter Uveitis Steroid Treatment (MUST) Trial Research Group1, Kempen JH, Altaweel MM, Holbrook JT, Jabs DA, et al. (2011) Randomized comparison of systemic anti-inflammatory therapy versus fluocinolone acetonide implant for intermediate, posterior, and panuveitis: the multicenter uveitis steroid treatment trial. Ophthalmology 118: 1916-1926. [Crossref]

12. Sangwan VS, Pearson PA, Paul H, Comstock TL (2014) Use of the fluocinolone acetonide intravitreal implant for the treatment of noninfectious posterior uveitis: 3 year results of a randomized clinical trial in a predominantly Asian population. Ophthalmol Ther 4: 1-9. [Crossref]
13. Boyer DS, Yoon YH, Belfort R Jr, Bandello F, Maturi RK, et al. (2014) Three-year, randomized, sham-controlled trial of dexamethasone intravitreal implant in patients with diabetic macular edema. Ophthalmology 121: 1904-1914. [Crossref]

14. Kiddee W, Trope GE, Sheng L, Beltran-Agullo L, Smith M, et al. (2013) Intraocular pressure monitoring post intravitreal steroids: a systematic review. Surv Ophthalmol 58: 291-310. [Crossref]

Copyright: (C2018 Schultz C. This is an open-access article distributed under the terms of the Creative Commons Attribution License, which permits unrestricted use, distribution, and reproduction in any medium, provided the original author and source are credited. 